\title{
O paradoxo da função de partição
}

\author{
The paradox of the partition function
}

\author{
J. Ricardo de Sousa ${ }^{* 1,2}$ \\ ${ }^{1}$ Universidade Federal do Amazonas, Departamento de Física, CEP 69077-000, Manaus, AM, Brasil. \\ ${ }^{2}$ Universidade Federal do Amazonas, Instituto Nacional de Ciência e Tecnologia de Sistemas Complexos, CEP 69077-000, \\ Manaus, AM, Brasil.
}

Recebido em 14 de março de 2021. Revisado em 28 de abril de 2021. Aceito em 04 de maio de 2021.

\begin{abstract}
A função de partição eletrônica para o átomo de hidrogênio será analisada, onde discutiremos o velho problema do paradoxo na mecânica estatística: a aparente divergência da função de partição, analisada primeiramente por Herzfeld em 1913. Este problema não tem sido discutido na maioria dos livros-texto de mecânica estatística, uma vez que não tem grande relevância no regime de baixas temperaturas (i. e., $k_{B} T \ll \varepsilon_{o} \simeq 13,6 \mathrm{eV}$, onde $\varepsilon_{o}$ é a energia de ionização). No regime de altas temperaturas (da ordem de $5000 \mathrm{~K}$ ), esta contribuição é importante nos estudos da física dos plasmas, espectroscopia e astrofísica. Apresentaremos alguns métodos de truncamento da função de partição a fim de deixar a série finita, analisando o comportamento do calor específico. Observamos que devido ao truncamento da série, o sistema terá níveis finitos e será refletido no comportamento tipo Schottky no calor específico.
\end{abstract}

Palavras-chave: divergência da função de partição, calor específico, átomo de hidrogênio.

The electronic partition function for the hydrogen atom will be analyzed, where we will discuss the old problem of the paradox in statistical mechanics: the apparent divergence of the partition function, first analyzed by Herzfeld in 1913. This problem has not been discussed in most textbooks statistical mechanics, since it has little relevance in the low temperature regime (i. e., $k_{B} T \ll \varepsilon_{o} \simeq 13,6 \mathrm{eV}$, where $\varepsilon_{o}$ is the ionization energy). In the high temperature regime (of the order of $5000 \mathrm{~K}$ ), this contribution is important in the studies of plasma physics, spectroscopy and astrophysics. We will present some methods of truncating the partition function in order to leave the finite series, analyzing the behavior of the specific heat. We observe that due to the series truncation, the system will have finite levels and will be reflected in the Schottky type behavior in the specific heat.

Keywords: partition function divergence, specific heat, hydrogen atom.

\section{Introdução}

O objetivo da disciplina mecânica estatística (graduação ou pós-graduação) é estudar os ensembles estatísticos [1], fazendo a conexão com a termodinâmica e aplicandoos na descrição de diversos sistemas na natureza (gás, líquido, sólido, plasma, etc). No caso particular do ensemble canônico temos um conjunto de sistemas idênticos macroscopicamente (macroestado), com $N$ partículas, dentro de um recipiente de volume $V$ num banho térmico à temperatura $T$, mas em diferentes estados microscópicos de energia (microestado). Neste caso, a energia flutua em torno do seu valor médio $E=\langle\mathcal{H}\rangle$, onde $\mathcal{H}$ é o Hamiltoniano do sistema (modelo), de modo que, a probabilidade de encontrar o sistema no estado de energia $E_{r}$ é dada por

$$
P\left(E_{r}\right)=\frac{\exp \left(-\beta E_{r}\right)}{Z},
$$

sendo

$$
Z=\operatorname{Tr}[\exp (-\beta \mathcal{H})]
$$

\footnotetext{
*Endereço de correspondência: jsousa@ufam.edu.br
}

a função de partição (que em alemão é Zustandssumme), onde $\operatorname{Tr}(\ldots)$ representa o traço de um operador (matriz) e $E_{r}$ é a autoenergia obtida a partir da solução da equação de Schrödinger independente do tempo.

Qualquer livro introdutório de mecânica estatística [10] contém a solução exata de quatro problemas fundamentais: partículas livres (modelo do gás ideal), osciladores harmônico desacoplados, sistema de spins localizados (não interagentes) na presença de um campo magnético (paramagnetismo) e o rotor rígido.

O primeiro problema tradicional ao estudar o ensemble canônico é o cálculo das propriedades termodinâmicas de um gás ideal monoatômico, cujo Hamiltoniano é dado por

$$
\mathcal{H} \equiv \mathcal{H}(\vec{p}, \vec{n})=\frac{1}{2 m} \sum_{i=1}^{N} \vec{p}_{i}^{2}+\sum_{i=1}^{N} \varepsilon_{n_{i}},
$$

onde $m$ é a massa de cada átomo, $\varepsilon_{n_{i}}$ é o espectro de energia (interna) do $i$-ésimo átomo, $\vec{p} \equiv\left(\vec{p}_{1}, \vec{p}_{2}, \ldots \vec{p}_{N}\right)$ são os momentos e $\vec{n} \equiv\left(n_{1}, n_{2}, \ldots n_{N}\right)$ o conjunto de números quânticos dos átomos.

O primeiro termo da Eq. (3) é a energia cinética (graus de liberdade de translação) dos átomos, enquanto 
o último termo o graus de liberdade interno dos átomos, que geralmente é ignorado nos livros-textos de mecânica estatística [1 10]. A presença deste segundo termo torna o sistema mais interessante e real, e veremos que trará problemas matemáticos na divergência da função de partição.

O ponto de partida no ensemble canônico é o cálculo da função de partição, que para sistemas clássicos é definida por

$$
Z(T, V, N)=\sum_{\vec{n}} g_{\vec{n}} \int \frac{\exp (-\beta \mathcal{H}) d^{3 N} \vec{r} d^{3 N} \vec{p}}{h^{3 N}}
$$

sendo $g_{\vec{n}}=\prod_{i=1}^{N} g_{n_{i}}, g_{n_{i}}$ é a degenerescência associada a energia $\varepsilon_{n_{i}}$ do $i$-ésimo átomo, onde usamos as notações:

$$
\left\{\begin{array}{l}
d^{3 N} \vec{r} \equiv \prod_{i=1}^{N} d^{3} \overrightarrow{r_{i}}=\prod_{i=1}^{N} d x_{i} d y_{i} d z_{i} \\
d^{3 N} \vec{p} \equiv \prod_{i=1}^{N} d^{3} \overrightarrow{p_{i}}=\prod_{i=1}^{N} d p_{x i} d p_{y i} d p_{z i}
\end{array} .\right.
$$

Como as moléculas são indistinguíveis, a Eq. (4) deve ser dividida pelo fator $N$ !. Devido ao Hamiltoniano (3) representar um sistema de $N$ partículas não interagentes, ao substituir na Eq. (4), ficaremos com

$$
Z(T, V, N)=\frac{1}{N !}\left[Z_{1 t}(T, V) \times Z_{o}(T)\right]^{N},
$$

sendo

$$
Z_{1 t}(T, V)=\int \frac{\exp \left(-\frac{\beta \vec{p}^{2}}{2 m}\right) d^{3} \vec{r} d^{3} \vec{p}}{h^{3}}=V\left(\frac{2 \pi m k_{B} T}{h^{2}}\right)^{3 / 2}
$$

a função de partição (uma partícula) associada aos graus de liberdade da energia de translação do átomo, e

$$
Z_{o}(T)=\sum_{n} g_{n} \exp \left(-\beta \varepsilon_{n}\right)
$$

é a função de partição eletrônica associada aos graus de liberdade da energia interna de um átomo com espectro de energia $\varepsilon_{n}$.

No caso de uma molécula diatômica (e poliatômica), a função de partição interna, $Z_{o}$, será o produto da função de partição rotacional, $Z_{o r}$, vibracional, $Z_{o v}$, e eletrônica, $Z_{o e}$, isto é, $Z_{o}=Z_{o r} \times Z_{o v} \times Z_{o e}$. No presente trabalho nos limitaremos ao estudo do gás monoatômico, desta maneira, a função de partição interna é dada apenas pela parte eletrônica, conforme expressa pela Eq. (8). Note que a função de partição (8) apresenta, a priori, somente o espectro de energia dos estados ligados do átomo, ou seja, para energia menor do que a energia de ionização do átomo.

Caso analisemos as propriedades termodinâmicas em altas temperaturas, ao ionizar o átomo devemos levar em consideração, ainda, os termos da função de partição dos elétrons livres (Fermi-Dirac), $Z_{o F D}$, de configuração,
$Z_{o c}$, e de radiação, $Z_{\text {or }}$. Estes termos na função de partição são fundamentais ao estudar as propriedades termodinâmicas no plasma e em sistemas estelares, onde temos altíssimas temperaturas e modelos estatísticos são propostos para simular as medidas experimentais 11. Vamos nos limitar, neste trabalho, a tratar do gás monoatômico em altas temperaturas sem que atinja valores de energia para ionizar os átomos, logo, restringiremos ao cálculo da função de partição eletrônica (8).

A conexão com a termodinâmica [10] é feita através da energia livre de Helmholtz (equação fundamental), definida por

$$
F(T, V, N)=-k_{B} T \ln Z(T, V, N) .
$$

Da equação de estado, substituindo (6) em (9), obtemos a equação de Clapeyron

$$
P=-\left(\frac{\partial F}{\partial V}\right)_{T, N} \rightarrow P V=N k_{B} T,
$$

que é independente da função de partição eletrônica (8).

Por outro lado, o cálculo da entropia, e, consequentemente, o calor específico, depende fortemente da função de partição eletrônica, que são expressos por

$$
\begin{aligned}
S(T, V, N)= & -\left(\frac{\partial F}{\partial T}\right)_{V, N} \\
= & S_{t}(T, V, N) \\
& +N k_{B}\left[\ln \left(Z_{o}(T)\right)+T \frac{\partial \ln Z_{o}(T)}{\partial T}\right],
\end{aligned}
$$

e

$$
\begin{aligned}
c_{V}(T) & =\frac{T}{N}\left(\frac{\partial S}{\partial T}\right)_{V, N} \\
& =\frac{3}{2} k_{B}+k_{B} T \frac{\partial}{\partial T}\left[\ln \left(Z_{o}(T)\right)+T \frac{\partial \ln Z_{o}(T)}{\partial T}\right],
\end{aligned}
$$

sendo $S_{t}(T, V, N)$ a entropia do gás ideal associada aos graus de liberdade de translação (fórmula de SackurTetrode) dada por

$$
\begin{aligned}
S_{t}(T, V, N)= & N k_{B}\left[\frac{3}{2} \ln \left(\frac{4 \pi m}{3 h^{2}}\right)\right. \\
& \left.+\ln \left(\frac{V}{N}\right)+\frac{3}{2} \ln \left(\frac{3}{2} k_{B} T\right)+\frac{5}{2}\right]
\end{aligned}
$$

A princípio, a função de partição eletrônica, $Z_{o}(T)$, não é relevante para o cálculo das propriedades termodinâmicas em baixas temperaturas. Entende-se como baixa temperatura quando a energia térmica $\left(k_{B} T\right)$ é muito menor do que a energia de ionização do átomo $\left(\varepsilon_{I}\right)$, que corresponde a temperatura, por exemplo, da ordem da temperatura ambiente $(\sim 300 \mathrm{~K})$. Isto justifica o fato de considerar apenas o primeiro termo (estado fundamental) na expansão (8), de modo que $Z_{o}(T)$ será uma 
constante (levamos em consideração a degenerescência do spin do elétron e a energia do estado fundamental nula).

Por outro lado, em altas temperaturas $\left(k_{B} T \approx \varepsilon_{I}\right)$, os estados excitados têm que ser levados em consideração e isto terá como consequência matemática a divergência da série da função de partição (8). O cálculo da função de partição e suas propriedades termodinâmicas de espécies atômicas (e moleculares) tem recebido muita atenção devido as diversas aplicações em física do plasma [12 15], dinâmica de fluidos [16, 17], espectroscopia [18] 21] e astrofísica [22 24. As duas principais dificuldades são encontrar o espectro de energia dos átomos (apenas o átomo de hidrogênio tem solução exata da equação de Schrödinger) e um critério satisfatório para o número quântico de corte para eliminar a divergência.

Neste trabalho iremos investigar a importância da função de partição eletrônica (8) sobre as propriedades termodinâmicas. Na seção 2, analisaremos como este termo influencia nas propriedades termodinâmicas, discutindo os aspectos históricos e operacionais, em especial investigaremos o problema da divergência da função de partição. Na seção 3, uma analogia com um sistema de dois níveis será analisada, e, finalmente, na seção 4 apresentaremos os comentários gerais.

\section{Função de Partição Eletrônica}

Vamos agora analisar a função de partição eletrônica, detendo-se para o caso de um gás ideal formado por moléculas de átomos de hidrogênio, que é dada por

$$
\begin{aligned}
Z_{o} & =\sum_{n} g_{H, n} \exp \left(-\beta \varepsilon_{H, n}\right) \\
& =2 \exp \left(-\beta \varepsilon_{o}\right) \sum_{n=1}^{\infty} n^{2} \exp \left(\beta \varepsilon_{o} / n^{2}\right) \\
& =2+2 \exp \left(-\beta \varepsilon_{o}\right) \sum_{n=2}^{\infty} n^{2} \exp \left(\beta \varepsilon_{o} / n^{2}\right),
\end{aligned}
$$

onde usamos o fato de que $g_{H, n}=2 n^{2}$ e $\varepsilon_{H, n}=\varepsilon_{o}(1-$ $\left.\frac{1}{n^{2}}\right)\left(\varepsilon_{o}=13,6058 \mathrm{eV}\right)$ para o átomo de hidrogênio. A justificativa para escrever os níveis de energia deslocados da energia de ionização, deve-se ao fato de tornar finita a função de partição em baixas temperaturas, isto é, $Z_{o}(T=0)=2$.

\subsection{Análise da divergência}

Matematicamente falando, mostraremos que o segundo termo da Eq. (14) apresenta a soma divergente, e, consequentemente, a função de partição eletrônica será infinita! Esta divergência na função de partição do átomo hidrogênio (ou átomos hidrogenóides) foi tema de grandes discussões na literatura, que certamente é falho, uma vez que obtemos teoricamente as propriedades termodinâmicas do gás monoatômico usando a mecânica estatística clássica, sem que esta divergência seja levada em consideração. Quase nenhum livro-texto de mecânica estatística trata deste tema, onde citamos os livros do Fowler e Tolman [25, 32] que apresentam uma breve discussão. A seguir mostraremos alguns argumentos para possibilidade da convergência desta série.

Vamos primeiro analisar alguns termos do somatório (14). Por exemplo, para o primeiro estado excitado $(n=2)$ temos $\varepsilon_{H, 2}=10.20 \mathrm{eV}$ e o gás numa temperatura ambiente $T=298 \mathrm{~K}\left(25^{\circ} \mathrm{C}\right)$ corresponde a energia térmica $k_{B} T=2.6 \times 10^{-2} \mathrm{eV}$. Desta maneira, o segundo termo do somatório é insignificante, da ordem de $\exp \left(-\beta \varepsilon_{H, 2}\right)=e^{-397} \simeq 10^{-172}$. Para altas ordens dos níveis, a energia tende para a energia de ionização (módulo) $\varepsilon_{o}=13,6 \mathrm{eV}$ e a exponencial tende para o valor $e^{-529} \simeq 10^{-230}$. Supondo agora uma temperatura mais moderada de $T=10^{3} \mathrm{~K}$, na Tabela 1 colocamos as primeiras contribuições da função de partição.

Como resultado dos valores da tabela 1, a função de partição será dada por

$$
Z_{o}=2+2.98 \times 10^{-51}+1.98 \times 10^{-60}+\ldots \simeq 2 .
$$

A razão entre a população do primeiro estado excitado e o estado fundamental é

$$
\frac{n_{2}}{n_{1}}=\frac{g_{H, 2}}{g_{H, 1}} \exp \left[-\left(\varepsilon_{H, 2}-\varepsilon_{H, 1}\right) k_{B} T\right]=1.5 \times 10^{-51},
$$

mostrando que apenas o estado fundamental é populado significantemente, justificando o valor da função de partição (15).

Apesar dos termos na exponencial serem pequenos, o fator da degenerescência $\left(\sim n^{2}\right)$ cresce muito rapidamente e isto resultará na divergência da série! A função de partição (14) satisfaz a seguinte desigualdade:

$$
Z_{o}>2+2 \times e^{-529} \sum_{n=2}^{\infty} n^{2} .
$$

Provaremos a seguir que a série acima é divergente, para isto usamos o fato de que

$$
K(\alpha, m) \equiv \sum_{n=1}^{m} \exp (-n \alpha)=\frac{1-\exp (-m \alpha)}{\exp (\alpha)-1}
$$

$\log \mathrm{O}$

$$
\sum_{n=1}^{m} n^{2}=\left.\frac{\partial^{2} K}{\partial \alpha^{2}}\right|_{\alpha=0}=\frac{1}{3} m^{3}+\frac{1}{2} m^{2}+\frac{1}{6} m .
$$

Tabela 1: Valores dos níveis de energia $\varepsilon_{H, n}$, degenerescência $g_{H, n}$ e parte da contribuição da função de partição $Z_{o}$ para uma temperatura $T=10^{3} \mathrm{~K}$.

\begin{tabular}{lccc}
\hline$n$ & $\varepsilon_{H, n} / \varepsilon_{o}$ & $g_{H, n}$ & $g_{H, n} \exp \left(-\varepsilon_{H, n} / k_{B} T\right)$ \\
\hline 1 & 0 & 2 & 2 \\
2 & $3 / 4$ & 8 & $2.98 \times 10^{-51}$ \\
3 & $8 / 9$ & 18 & $1.98 \times 10^{-60}$ \\
$\ldots$ & $\ldots$ & $\ldots$ & $\ldots$ \\
$\ldots$ & $\ldots$ & $\ldots$ & $\ldots$ \\
$n>>1$ & 1 & $2 n^{2}$ & $2 n^{2}\left(2.59 \times 10^{-69}\right)$ \\
\hline
\end{tabular}


Portanto, mesmo se todos os termos da expansão (14 forem multiplicados por $10^{-230}$, a soma total diverge na forma assintótica com $m^{3}$ quando $m \rightarrow \infty$. Como consequência da divergência da função de partição, a probabalidade de encontrar o átomo de hidrogênio em temperatura finita no estado fundamental é nula $\left(p_{1}=\right.$ $2 / Z \rightarrow 0)$, ou seja, em qualquer temperatura $T>0 \mathrm{~K}$ o átomo de hidrogênio poderia estar nos estados excitados. Certamente, isto não está correto, alguma coisa está errada na análise desta série, Eq. (14).

Para átomos diferentes (e moléculas) é esperado também que o espectro de energia (não solúvel exatamente) apresente infinitos estados ligados e encontramos o mesmo resultado (paradoxal) da função de partição divergente. Isto é inerente a qualquer sistema com interação coulombiana, aqui detalhamos o hidrogênio, que é o elemento mais abundante no Universo, onde as suas propriedades termodinâmicas são muito conhecidas.

\subsection{Ponto de corte no número quântico}

O problema da divergência na função de partição eletrônica foi primeiramente apontado pelo físico austríaco Karl Herzfeld [27], e as vezes é conhecida na literatura como o problema da divergência de Herzfeld. Em baixas temperaturas $\left(k_{B} T \ll \varepsilon_{o}\right)$ foi considerado apenas o termo do estado fundamental na soma, de modo que $Z_{o}=2$. Apesar de não ter sido justificado matematicamente esta aproximação, isto tem sido usado constantemente nos livros-textos 2 10. Aqui exploraremos a validade desta aproximação, usando argumentos físicos. Para altas temperaturas $\left(k_{B} T \simeq \varepsilon_{o}\right)$, os estados excitados não podem ser ignorados, por exemplo, para o átomo de hidrogênio em $T=25.000 \mathrm{~K}$, os estados excitados contribuem com $4 \%$ para $Z_{o}$, e esta contribuição cresce com o aumento da temperatura.

Sendo a energia de ionização do átomo de hidrogênio $\left(\varepsilon_{I}=\varepsilon_{o}=13,6 \mathrm{eV}\right)$ maior do que muitos dos outros átomos (apenas os gases nobres são maiores), a dependência com a temperatura de $Z_{o}(T)$ será mais relevante para os outros átomos. Os piores casos de átomos com baixa energia de ionização são os metais alcalinos, seguidos pelos alcalinos terrosos e elementos de transições. Para o sódio, o problema se torna sério em torno de $5000 \mathrm{~K}$, onde o calor específico atinge um ponto de máximo. Para os elementos de transição e terras raras existem um problema adicional da existência de muitos estados de baixa energia, que torna contribuições significantes mesmo em baixa temperatura.

Para obtermos $Z_{o}(T)$ finita, válida para toda temperatura, um método razoável é simplificar a soma através de um ponto de corte no número quântico, isto é, a soma realizada até um valor máximo $n=n_{\max }$. Este problema do truncamento da série da função de partição (14) tem uma longa história, iniciado com Fermi [28, Ecker 29, 30, Margenau e Lewis 31, Fowler 32 e outros pesquisadores $33-40$, e com os trabalhos de Drellishak 41] e Capitelli e colaboradores 42 45] completando os níveis de energia inexistentes nas tabelas de Moore [4648. para os átomos livres.

Para entender este paradoxo, recorremos ao tamanho do orbital eletrônico, e consideremos ser bem aproximado pelos raios de Bohr, que é dado por

$$
r_{H, n}=n^{2} a_{o}
$$

onde $a_{o}=0,53 \times 10^{-10} \mathrm{~m}$ é o raio (de Bohr) do estado fundamental do átomo de hidrogênio.

Se escolhermos $n=10^{5}$, teremos um átomo de tamanho macroscópico $(\approx 0,53 \mathrm{~m})$. Isto sugere que a função de onda do elétron interage com o recipiente que contém o átomo, portanto, a função de onda não poderia ser do átomo de hidrogênio. Desta maneira, o elétron no átomo de hidrogênio, nestas condições de grandes números quânticos, será melhor descrito por funções de onda de uma partícula numa caixa de potencial infinito. É bem conhecido que a energia dos estados excitados nesta situação cresce $\operatorname{com} n^{2}$ (i. e., $\varepsilon_{n} \simeq n^{2}$ ). Consequentemente, o fator exponencial na função de partição decresce muito rapidamente e o termo genérico na série tende a zero (para $n>10^{5}$ é da ordem de $\left.a_{n} \simeq n^{2} \exp \left(-n^{2}\right) \rightarrow 0\right)$.

Um cálculo numérico, resolvendo a equação de Schrödinger para o átomo de hidrogênio dentro de uma caixa esférica de raio $\delta$, foi realizado por Capitelli e Giordano 49, onde mostraram que os últimos níveis de energia (por exemplo, $n>28$ para $\delta=10^{3} a_{o}$ ) convergem para os níveis de energia da caixa esférica, isto é, $\varepsilon_{n} \simeq n^{2}$. Isto, a priori, justifica a presença de um ponto de corte para o número quântico no somatório da função de partição.

Podemos estimar a contribuição dos níveis excitados introduzindo um corte (valor máximo de $n_{\max }$ ) no somatório. Suponhamos que $n_{\max }=10^{5}$ e limitamos os termos do somatório pelo fator exponencial $\exp \left(-\beta \varepsilon_{o}\right) \simeq$ $10^{-230}$, usando o resultado 17 com $n=n_{\max }$, obtemos

$$
\begin{aligned}
Z_{o} & \simeq 2+2 \times 10^{-230} \sum_{n=1}^{m} n^{2} \\
& \simeq 2+2 \times 10^{-230}\left[\frac{1}{3}\left(10^{5}\right)^{3}+\ldots\right] \\
& \simeq 2+2 \times 10^{-215} \simeq 2,
\end{aligned}
$$

logo, a única contribuição para a função de partição eletrônica é devido ao estado fundamental $(n=1)$, portanto, a probabilidade de encontrar neste estado é um.

\subsection{Critérios do ponto de corte para o número quântico}

A medida que o número quântico principal $n$ aumenta, o raio atômico cresce segundo a lei de potência 20 para o caso do átomo de hidrogênio, mas de uma maneira geral, os átomos (moléculas) têm o seu tamanho aumentado a medida que excitamos. Então é esperado que o raio 
atômico não exceda a dimensão macroscópica, de modo que estipulasse existir um valor máximo $n_{\max }$ para o número quântico.

Inicialmente, foram propostos dois critérios: o proposto por Fermi (critério de Fermi) [28, no qual o raio do átomo não exceda a distância média interatômica $\left(\sim(V / N)^{1 / 3}=v^{1 / 3}\right.$, onde $V$ é o volume do recipiente que contém o gás e $N$ o número de moléculas do gás), ou seja (aproximação do volume excludente),

$$
a_{o} n_{\max }^{2}=v^{1 / 3},
$$

e o critério de Griem [50], expresso em função do comprimento de Debye $(D)$ dado por

$$
a_{o} n_{\max }^{2}=D \text {. }
$$

O comprimento de Debye $(D)$ fornece uma medida da distância na qual a influência de um campo elétrico perturbativo é sentida no interior do plasma. A partir da equação de Poisson, podemos mostrar que o potencial sentido no interior do plasma tem a forma $\phi(x)=\phi(0) \exp (-x / D)$, onde $x$ é a distância ao longo do plasma a partir de uma origem que tem a presença da perturbação $\phi(0)$ e $D=\sqrt{\frac{k_{B} T}{4 \pi e^{2} \rho_{p}}}$, sendo $\rho_{p}$ a densidade do plasma (varia entre $10^{9}$ a $10^{33}$ partículas carregadas $/ \mathrm{m}^{3}$ das nebulosas ao centro do Sol, respectivamente).

Os dois critérios (ad hoc) são aplicados em geral para obter as propriedades do plasma em baixa pressão. No critério de Fermi, o valor de $n_{\max }$ depende da temperatura e pressão $(T, P)$ e o critério do Griem da temperatura e densidade $(T, \rho)$. Para um átomo dentro de uma caixa esférica de raio $\delta$, do critério de Fermi 22 , obtemos

$$
n_{\max }=\left(\frac{4 \pi}{3}\right)^{1 / 6} \sqrt{\frac{\delta}{a_{o}}}=1.27 \sqrt{\frac{\delta}{a_{o}}} .
$$

Escolhendo os valores $\delta / a_{o}=10^{3}$ e $10^{4}$, da relação (24) encontramos $n_{\max }=40$ e 127 , respectivamente. Simulando o átomo de hidrogênio no interior de uma caixa esférica de raio $\delta$, a equação de Schrödinger no estado $l=0$ foi resolvida numericamente, com as condições de contorno para a solução radial $R(r=0)=$ $R(r=\delta)=0$, Capitelli e Giordano [49] mostraram que $n_{\max }=28$ e 89 , respectivamente, para os valores das razões $\delta / a_{o}=10^{3}$ e $10^{4}$. Foi mostrado também, que estes valores para $n_{\max }$ não são alterados quando $l>0$. No caso do modelo de Fermi, a função de partição (14) é truncada no número quântico $n_{\max }$. Por outro lado, no caso do modelo de Capitelli e Giordano (CG), temos um espectro de energia de estados ligados $\varepsilon<0$ para $n<n_{\max }$ e estados não ligados $\varepsilon>0$ para $n>n_{\max }$, ou seja,

$$
\varepsilon_{H, n}=\left\{\begin{array}{l}
\varepsilon_{o}\left(1-\frac{1}{n^{2}}\right), n<n_{\max } \\
\frac{\varepsilon_{o} \pi^{2} n^{2}}{q^{2}}, n>n_{\max }
\end{array},\right.
$$

onde $q=\delta / a_{o}$.
Note que para $n>n_{\max } \simeq 0.9 q^{1 / 2}$ o espectro de energia assemelha-se a de uma partícula livre numa caixa, isto não significa que o átomo foi ionizado, mas sim devido ao efeito do volume finito temos a mudança de comportamento do espectro de energia. O valor do ponto de corte $n_{\max }$ no modelo F, Eq. (24), é maior do que o valor encontrado no modelo $\mathrm{CG}$.

Fazendo o truncamento da série (14), obtemos a função de partição aproximada dada por

$$
Z_{o}(T)=2+2 \exp \left(-\beta \varepsilon_{o}\right) \sum_{n=2}^{n_{\max }} n^{2} \exp \left(\beta \varepsilon_{o} / n^{2}\right),
$$

onde $n_{\text {max }}$ depende de cada modelo estudado, que é uma função do fator $q=\delta / a_{o}$.

As funções de partições $Z_{o}^{C G}(T)$ e $Z_{o}^{F}(T)$ são funções monotonicamente crescente com a temperatura, o que corresponde ao resultado matematicamente esperado 42 45. Em $T=0$, ambos os modelos encontram $Z_{o}^{F, C G}(0)=2$ e no limite $T \rightarrow \infty$, devido ao truncamento do número quântico, usando o resultado (19), obtemos um valor finito para $\delta / a_{o}=10^{3}\left(10^{4}\right)$ que é dado por

$$
\begin{aligned}
Z_{o}^{F, C G}(T \rightarrow \infty) & =\frac{1}{3} n_{\max }^{3}+\frac{1}{2} n_{\max }^{2}+\frac{1}{6} n_{\max } \\
& =\left\{\begin{array}{l}
7714(238965), \text { modelo } \mathrm{CG} \\
22140(690880), \text { modelo } \mathrm{F}
\end{array}\right.
\end{aligned}
$$

Em particular, para $q=\delta / a_{o}=10^{3}$, encontramos $n_{\max }=28$ (40) para o modelo $\mathrm{CG}(\mathrm{F})$, substituindo na Eq. 26 temos $Z_{o}^{C G}(T) \leq Z_{o}^{F},(T)$, que é compreendido por conter menos estados na função de partição no modelo CG. Usando a expressão $(12)$, realizando a soma numericamente para cada valor da temperatura, obtemos o calor específico $c_{V}(T)$. Em baixas temperaturas $\left(k_{B} T / \varepsilon_{o} \lesssim 0.06\right)$, prevalece apenas os graus de liberdade do movimento de translação, resultando no valor $c_{V}=3 k_{B} / 2$, enquanto que em temperatura intermediária $\left(0.06 \lesssim k_{B} T / \varepsilon_{o} \lesssim 0.2\right)$ os graus de liberdade interno (eletrônico) tornam-se significantes, contribuindo para o valor do calor específico, apresentando um comportamento tipo Schottky, característico de um sistema constituído por dois níveis. Em altas temperaturas $\left(k_{B} T / \varepsilon_{o} \gtrsim 0.2\right)$, os níveis discretos de energia tornam-se insignificantes e a função de partição eletrônica não contribui para o calor específico, resultando, assim, no mesmo valor clássico $c_{V}=3 k_{B} / 2$.

Na Figura 1 apresentamos os comportamentos dos calores específico em função da temperatura reduzida $k_{B} T / \varepsilon_{o}$ obtidas pelos dois modelos $\mathrm{F}$ e $\mathrm{CG}$, e na figura inserida temos as respectivas funções de partições eletrônicas. Este comportamento tipo Schottky do calor específico foi observado experimentalmente em plasmas de gás de hidrogênio 42 45].

Aplicamos também o método CG levando em consideração a região do espectro de energia positiva (25), mas o resultado foi mostrado insatisfatório, mostrando 


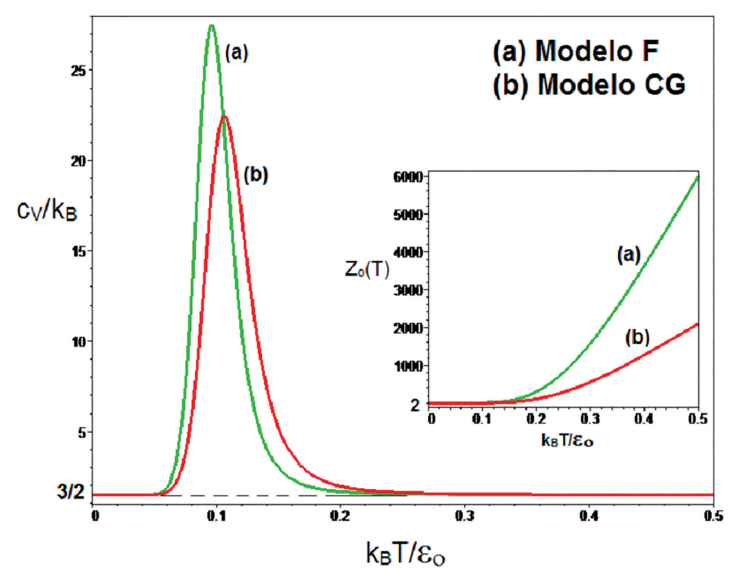

Figura 1: Comportamento do calor específico eletrônico, adicionado o valor do calor específico do grau de liberdade de translaçcão $\left(c_{t V}=3 k_{B} / 2\right)$, normalizado $c_{V} / k_{B}$ em função da temperatura reduzida $k_{B} T / \varepsilon_{o}$ para os modelos Fermi (a) e Capitelli e Giordano (b). Na figura inserida mostramos os comportamentos das respectivas funções de partição.

um comportamento do calor específico diferente do apresentado na Figura 1. Existem outros critérios fixando apenas um valor para $n_{\max }$, não dependendo de nenhum parâmetro termodinâmico, calculando diretamente a função de partição para uma série truncada 31. Outros métodos usam apenas o critério da energia térmica (i. e., $\left.\varepsilon_{H, n}<\varepsilon_{o}-k_{B} T\right)$, por exemplo, o discutido por McChesney [52. Este método do truncamento é muito apropriado para tratar plasmas, e tem sido recomendado por McBride e Gordon no programa da NASA [53.

\section{Modelo do Sistema de Dois Níveis}

Como proposta para decrever as propriedades termodinâmicas do gás de hidrogênio, Capitelli e colaboradores 42 45 associaram este gás a um sistema de dois níveis. Vamos primeiro revisar o problema de dois níveis com energia (degenerescência) $\varepsilon_{1}=0\left(g_{1}\right)$ e $\varepsilon_{2}\left(g_{2}\right)$, de modo que os números de ocupação são dados por

$$
\left\{\begin{array}{l}
n_{1}=\frac{N_{1}}{N}=\frac{g_{1}}{g_{1}+g_{2} \exp \left(-\beta \varepsilon_{2}\right)} \\
n_{2}=\frac{N_{2}}{N}=\frac{g_{2} \exp \left(-\beta \varepsilon_{2}\right)}{g_{1}+g_{2} \exp \left(-\beta \varepsilon_{2}\right)}
\end{array},\right.
$$

onde $N=N_{1}+N_{2}$ é o número total de partículas.

Definindo uma temperatura característica $T_{2} \equiv$ $\varepsilon_{2} / k_{B}$, os números de populações 28 nos limites de baixas e altas temperaturas valem

$$
\left\{\begin{array}{l}
T \ll T_{2} \rightarrow\left(N_{1}=N, N_{2}=0\right) \\
T \gg T_{2} \rightarrow\left(N_{1}=\frac{N g_{1}}{g_{1}+g_{2}}, N_{2}=\frac{N g_{2}}{g_{1}+g_{2}}\right)
\end{array}\right.
$$

ou seja, em baixas temperaturas, o estado fundamental é populado significantemente, enquanto em altas temperaturas os níveis são igualmente populados e proporcionais ao seu grau de degenerescência.
A energia média por partículas e os correspondentes limites de baixas e altas temperaturas são dadas por

$$
\begin{aligned}
u(T) & =n_{1} \varepsilon_{1}+n_{2} \varepsilon_{2} \\
& =\frac{g_{2} \varepsilon_{2} \exp \left(-\beta \varepsilon_{2}\right)}{g_{1}+g_{2} \exp \left(-\beta \varepsilon_{2}\right)} \\
& \rightarrow\left\{\begin{array}{l}
T \ll T_{2} \rightarrow u(T)=0 \\
T \gg T_{2} \rightarrow u(T)=\frac{g_{2} \varepsilon_{2}}{g_{1}+g_{2}}
\end{array} .\right.
\end{aligned}
$$

Em geral, temos $g_{2}>>g_{1}$ e $\varepsilon_{2} \simeq \varepsilon_{I}, \varepsilon_{I}$ sendo a energia de ionização. Como consequência, em altas temperaturas temos $u(T) \simeq \varepsilon_{I}$. Finalmente, o calor específico é dado por

$$
c(T)=\frac{d u(T)}{d T}=k_{B}\left(\frac{T_{2}}{T}\right)^{2} \frac{g_{1} g_{2} \exp \left(-T_{2} / T\right)}{\left[g_{1}+g_{2} \exp \left(-T_{2} / T\right)\right]^{2}},
$$

que tende a zero nos limites de baixas e altas temperaturas, apresentando um comportamento tipo Schottky. Os cálculos acima se encontram em qualquer livrotexto de mecânica estatística [2 10], apenas colocamos os principais resultados.

Como aplicação, vamos supor que o átomo de hidrogênio seja representado por um sistema de dois níveis: (i) o estado fundamental, caracterizado pela energia $\varepsilon_{1}=0$ e degenerescência $g_{1}=g_{H, 1}=2$ (onde $g_{H, n}=2 n^{2}$ é a degenerescência do átomo de hidrogênio) e (ii) um estado excitado tendo a degenerescência igual a soma das degenerescências e a energia igual a valor médio de todos estados excitados de $n=2$ até um certo valor máximo $n_{\max }$, isto é,

$$
\left\{\begin{array}{l}
g_{2}=\sum_{n=2}^{n_{\max }} g_{H, n}=2 \sum_{n=2}^{n_{\max }} n^{2} \\
\varepsilon_{2}=\frac{1}{g_{2}} \sum_{n=2}^{n_{\max }} g_{H, n} \varepsilon_{H, n}=\varepsilon_{o}\left[1-\frac{2\left(n_{\max }-1\right)}{g_{2}}\right]
\end{array},\right.
$$

onde $\varepsilon_{H, n}=\varepsilon_{o}\left(1-\frac{1}{n^{2}}\right)$ e $\varepsilon_{I}=\varepsilon_{o}=13,6058 \mathrm{~V}$ é a energia de ionização.

Usando (19) em (32), mostramos que

$$
g_{2}\left(n_{\max }\right)=\frac{1}{3} n_{\max }^{3}+\frac{1}{2} n_{\max }^{2}+\frac{1}{6} n_{\max }-2,
$$

é uma função monotonicamente crescente com o aumento de $n_{\max }$. A energia do estado excitado $\varepsilon_{2}$ e a temperatura característica $T_{2}=\varepsilon_{2} / k_{B}$, bem como o grau de degenerescência $g_{2}$ dependem de $n_{\max }$, como mostrado na Tabela 2 , onde $g_{2}$ e $T_{2}$ são dados para alguns valores de $n_{\max }$. A medida que $n_{\max }$ cresce, a temperatura característica $T_{2}$ tende assintoticamente ao valor $T_{2}^{*}=\varepsilon_{I} / k_{B}=157.888 \mathrm{~K}$ quando $n_{\max } \rightarrow \infty$. Neste limite, o número de ocupação 29 corresponde a $N_{1}=0$ e $N_{2}=N$, que significa todos os átomos de hidrogênio nos estados excitados $(n \geq 2)$.

Usando a expressão (31) com $g_{1}=2$ e $g_{2}$ dado pela Eq. (33), fixando um dado valor de $n_{\max }$, obtemos o calor específico em função da temperatura. Na Figura 2 
Tabela 2: Valores de $g_{2}$ e $T_{2}$ para alguns $n_{\max }$.

\begin{tabular}{lcc}
\hline$n_{\max }$ & $g_{2}$ & $T_{2}$ \\
\hline 5 & 108 & 146.187 \\
10 & 768 & 154.181 \\
25 & 11.048 & 157.701 \\
50 & 85.848 & 157.701 \\
75 & 286.898 & 157.800 \\
$\infty$ & $\infty$ & 157.888 \\
\hline
\end{tabular}

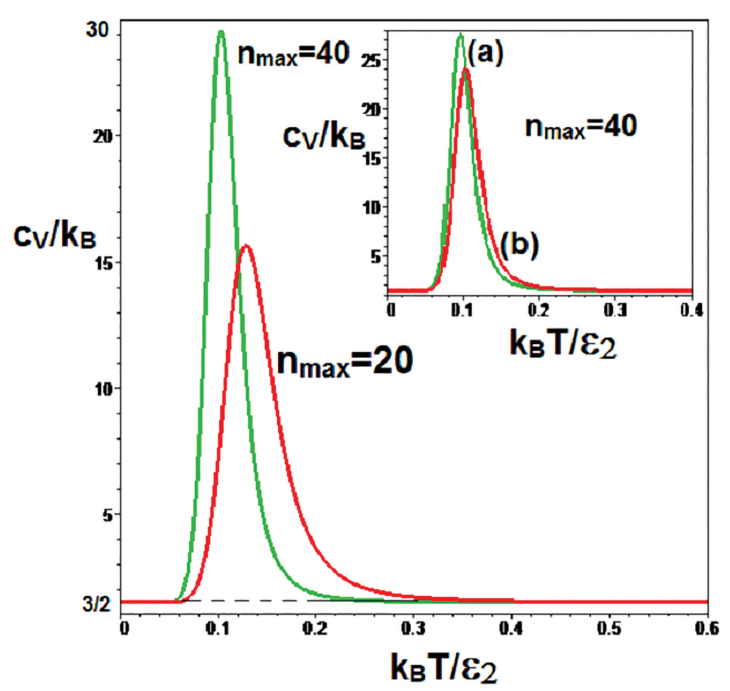

Figura 2: Comportamento do calor específico normalizado $c_{V} / k_{B}$ para um sistema de dois níveis, adicionado o valor do calor específico do grau de liberdade de translação $\left(c_{t V}=\right.$ $\left.3 k_{B} / 2\right)$, em função da temperatura reduzida $k_{B} T / \varepsilon_{2}=T / T_{2}$ simulando o gás de hidrogênio para dois valores de $n_{\max }$. A figura inserida corresponde a comparação entre os resultados dos modelos fazendo o truncamento da função de partição (a) e o modelo de dois níveis (b) para $n_{\max }=40$.

apresentamos o comportamento do calor específico, correspondente ao modelo de dois níveis adicionado o valor do calor específico do grau do movimento de translação $\left(c_{t V}=3 k_{B} / 2\right)$, em função da temperatura reduzida $k_{B} T / \varepsilon_{2}$. Escolhemos os valores $n_{\max }=20$ e 40 para nossa análise, onde observamos que a medida que $n_{\max }$ cresce, a temperatura do ponto de máximo e a largura da curva diminuem gradualmente. Para baixas e altas temperaturas, o calor específico é uma constante igual ao valor associado apenas ao grau de liberdade de translaçao (i. e., $c_{V}=3 k_{B} / 2$ ). Na figura inserida, comparamos os resultados obtidos pelo truncamento direto através da função de partição até o valor $n_{\max }=$ 40 (curva a) com o correspondente modelo de dois níveis (curva b).

O comportamento do calor específico das Figuras 1 e 2 são qualitativamente equivalentes, como esperado para um sistema de níveis finitos de energia $\left(n \leq n_{\max }\right)$. Note que este modelo é apenas uma descrição fenomenológica do truncamento da série da função de partição, onde as propriedades termodinâmicas em altas temperaturas do gás formado por átomos de hidrogênio são calculadas. A metodologia pode ser estendida para outros gases, e justifica o ponto de vista para o uso deste método do truncamento [54].

\section{Comentários Gerais}

Neste trabalho discutimos métodos de truncamentos para o cálculo da função de partição eletrônica, em particular estudamos um sistema de um gás formado por átomos de hidrogênio. O aparente paradoxo da divergência da função de partição eletrônica foi resolvida através de métodos de truncamentos. O método de Fermi, onde limita o tamanho do átomo igual a distância média entre os átomos $\left(\sim(V / N)^{1 / 3}\right)$, foi plenamente justificado através da simulação de um sistema com átomos de hidrogênio dentro de um volume finito esférico de raio $\delta$ resolvendo a equação de Schrödinger [49]. Este truncamento no valor do número quântico principal não limita o sistema a está no volume finito, apenas relaciona com a distância média entre os átomos. Uma outra metodologia usada, e proposta por Capitelli e colaboradores 42 45], foi a conexão do gás de hidrogênio através de um modelo de dois níveis. Todos estes métodos têm em comum os mesmos comportamentos das propriedades termodinâmicas, por exemplo, o calor específico numa região de temperatura moderada apresenta um ponto de máximo (comportamento tipo Schottky), enquanto que nos limites de baixas e altas temperaturas o calor específico é constante e igual a $3 k_{B} / 2$ (calor específico associado ao grau de liberdade do movimento de translação dos átomos). Este comportamento do calor específico foi observado no sistema de plasmas formado por hidrocarbonetos 55. Na parte II deste trabalho vamos apresentar métodos de regularização da função de partição, que não faz uso do truncamento, método este muito usado em teoria de campos.

\section{Agradecimentos}

O autor agradece aos professores Constantino Tsallis (CBPF) e Wagner Figueiredo (UFSC) pela leitura crítica, sugestões e incentivo em submeter este trabalho.

\section{REFERÊNCIAS}

[1] J.W. Gibbs, Elementary Principles in Statistical Mechanics (Yale University Press, New Haven, 1902).

[2] C. Kittel, Elementary Statistical Physics (John Wiley, New York, 1958).

[3] R. Kubo, Statistical Mechanics (Interscience Publishers, New York, 1965).

[4] L.D. Landau e E.M. Lifshitz, Statistical Physics (Pergamon Press, Oxford, 1958).

[5] A. Sommerfeld, Thermodynamic and Statistical Mechanics (Academic Press, New York, 1956).

[6] E. Schrodinger, Statistical Thermodynamics (Cambridge University Press, Cambridge, 1960). 
[7] G.S. Rushbrooke, Introduction to Statistical Mechanics (Clarendon Prss, Oxford, 1955).

[8] R.K. Pathria, Statistical Mechanics (Pergamon Press, Oxford, 1972).

[9] K. Huang, Statistical Mechanics (John Wiley, New York, 1987), $2^{a}$ ed.

[10] S. Salinas, Introdução à Física Estatística (EDUSP, São Paulo, 2013), $2^{a}$ ed.

[11] W. Ebeling, V.E. Fortov e V. Filinov, Quantum Statistics of Dense Gases and Nonideal Plasmas (Springer, Berlin, 2017).

[12] M. Capitelli, D. Giordano e G. Colonna, Phys. Plasmas 15, 082115 (2008).

[13] K. Singh, G. Singh e R. Sharma,Phys. Plasmas 17, 072309 (2010).

[14] R. Sharma, G. Singh e K. Singh, Phys. Plasmas 18, 083510 (2011).

[15] W.Z. Wang, M.Z. Rong, J.D. Yan, A.B. Murphy e J.W. Spencer, Phys. Plasmas 18, 113502 (2011).

[16] D. Bernardi, V. Colombo, G.G.M. Coppa e A. D'Angola, Eur. J. Phys. D 14, 337 (2001).

[17] G. Colonna, A. D'Angola e M. Capitelli, Phys. Plasmas 19, 072115 (2012).

[18] R. Gaudiuso, M. Dell'Aglio, O.D. Pascale, G. Senesi e A. De Giacomo, Sensors 10, 7434 (2010).

[19] L.J. Radziemski, Lasers-Induced Plasmas and Applications (CRC Press, Boca Raton, 1989).

[20] H. Hahn e N. Omenetto, Appl. Spectrosc 64, 335A (2010).

[21] C. Argón e J.A. Aguilera, Spectrochim. Acta 63, 893 (2008).

[22] D.G. Hummer e D. Mihalas, Astrophys. J. 331, 794 (1988).

[23] D. Mihalas, W. Daeppen e D.G. Hummer, Astrophys. J. 331, 815 (1988).

[24] W. Daeppen, D. Mihalas, D.G. Hummer e B.W. Mihalas, Astrophys. J. 332, 261 (1988).

[25] R.H. Fowler, Statistical Mechanics: The theory of the properties of matter in equilibrium (Cambridge University, Cambridge, 1936).

[26] R.C. Tolman, The Principles of Statistical Physics (Oxford University Press, London, 1938).

[27] K.F. Herzfeld, Sitzungsberichte der Königlichen Akademic der Wissenschaften 121, 593 (1912).

[28] E. Fermi,Z. Phys. 26, 54 (1924).

[29] G. Ecker e W. Weizel, Ann. Phys. 452, 126 (1956).

[30] G. Ecker e W. Kröll, Phys. Fluids 6, 62 (1963).

[31] H. Margenau e M. Lewis, Rev. Mod. Phys. 31, 569 (1959).

[32] R.H. Fowler, Philos. Mag. 1, 845 (1926).

[33] H.C. Urey, Astrophys. J. 49, 1 (1924).

[34] E.P. Wigner, Phys. Rev. 94, 77 (1954).

[35] R.E. Treves, Phys. Rev. 102, 1533 (1926).

[36] B.F. Gray, J. Chem. Phys. 36, 1801 (1962).

[37] S.J. Strickler, J. Chem. Ed. 43, 364 (1966).

[38] C.A. Rouse, Phys. Rev. 163, 62 (1967).

[39] A. Wehrl, Rev. Mod. Phys. 50, 221 (1978).

[40] M. Grabowski, Rep. Math. Phys. 23, 19 (1986).
[41] K.S. Drellishak, D.P. Aeschliman e A.B. Cambel, Phys.Fluids 8, 1590 (1965).

[42] M. Capitelli, G. Colonna, D. Giordano, L. Marraffa, A. Casavola, P. Minnelli, D. Pagano, L.D. Pietanza e F. Taccogna, Technical Report STR-246, European Space Agency (2005).

[43] M. Capitelli, G. Colonna, D. Giordano, L. Marraffa, A. Casavola, P. Minnelli, D. Pagano, L.D. Pietanza e F. Taccogna, J. Spacecr. Rockets 42, 980 (2005).

[44] M. Capitelli e E. Molinari, J. Plasma Phys. 4, 335 (1970).

[45] M. Capitelli, G. Colonna e A. D'Angola, Fundamental aspects of plasma chemical physics: Thermodynamics (Springer, Berlin, 2012).

[46] C.E. Moore, Atomic Energy Levels (Hidrogen through Vanadium) (U.S. Government Printing Office, Washington, 1949), v. 1.

[47] C.E. Moore, Atomic Energy Levels (Chromium through Niobium) (U.S. Government Printing Office, Washington, 1952), v. 2.

[48] C.E. Moore, Atomic Energy Levels (Molybdenum through Lanthanum and Hafnium through Actinium) (U.S. Government Printing Office, Washington, 1958), v. 3.

[49] M. Capitelli e D. Giordano, Phys. Rev. A 80, 032113 (2009).

[50] H.R. Griem, Principles of Plasma Spectroscopy (Cambridge University Press, Cambridge, 1997).

[51] M.R. Zaghloul, Phys. Lett. A 377, 119 (2013).

[52] M. McChesney, Can. J. Phys. 42, 2473 (1964).

[53] B.J. McBride e S. Gordon, NASA TN D-4097 (NASA, Washington, 1967).

[54] G. D'Ammando, G. Colonna e M. Capitelli, Phys. Plasmas 20, 032108 (2013).

[55] O. Askari, G.P. Beretta, K. Eisazadeh-Far e H. Metghalch, Eur. Phys. J. D 70, 159 (2016). 